\title{
Development of a Noble Field Emitter using Self-Aligned Carbon Nanotubes
}

\author{
K. Tatsumi,* T. Tanji, ${ }^{*}$ and M. Kusunoki** \\ * Department of Electronics, Nagoya University, Chikusa, Nagoya 464-8503, Japan \\ ** Japan Fine Ceramics Center, Mutsuno, Atsuta, Nagoya 456-8587, Japan
}

Recently, carbon nanotubes have attracted great interest because of their unique structural, electrical and mechanical properties, which include high aspect ratio, high mechanical strength, and chemical stability. In addition, applications such as field electron emitters, hydrogen storage media, and battery electrodes, which take advantage of these properties, are strongly expected. The present study examines the application of carbon nanotubes as field electron emitters, in particular, for an electron microscope. Various promising results for this application have been reported previously. Most of the carbon nanotubes examined were prepared by arc discharge, thermal chemical vapor deposition (CVD) or laser ablation, and they were mounted on tungsten filaments [1]. We herein report the field emission properties of carbon nanotubes produced by the surface decomposition of silicon carbide [2].

A field emitter tip in which the upper portion is coated with aligned carbon nanotubes is referred to as a carbon nanotube (CNT) tip. The procedure by which to produce a CNT tip from a SiC single crystal is described below.

First, a SiC single crystal is polished well to produce a smooth (000-1) surface because carbon nanotubes cannot grow on a rough surface. Then, a SiC tip (FIG.1) is mechanically cut out from a $\mathrm{SiC}$ single crystal by a precise diamond cutter which has a special blade with a rectangular wedge. At this stage, its top surface is a rectangle of $1 \mu \mathrm{m} \times$ a few $\mu \mathrm{m}$. The tip is then etched by FIB in order to reduce dimensions of the top to $0.3 \mu \mathrm{m} \times 0.3 \mu \mathrm{m}$, when the $(000-1)$ surface is protected by courting an aluminum thin layer instead of a tungsten layer.

After this process, the tip is heated at $1700 \mathrm{~K}$, for $6 \mathrm{hrs}$ in a vacuum of $1 \times 10^{-2} \mathrm{~Pa}$ using an electric resistance carbon heater. By surface decomposition of silicon carbide, aligned carbon nanotubes are preferably produced on the top perpendicular to the $\mathrm{SiC}(000-1)$ surface, and the $\mathrm{SiC}$ tip is changed into a CNT tip.

Finally, the CNT tip is heated again at $1700 \mathrm{~K}$, for $10 \mathrm{hrs}$ in a vacuum of $1 \times 10^{-4} \mathrm{~Pa}$. This procedure grows graphite layers and enhances the conductance of the CNT tip. The CNT tip is mounted on a tungsten filament, and its characteristic as a field emitter is examined.

Figure 2 is a TEM image of the top of a CNT tip. We can see that aligned carbon nanotubes having a diameter of 5 or $6 \mathrm{~nm}$ and a length of approximately $100 \mathrm{~nm}$ have grown on the top of the tip.

Figure 3 shows the emission current against the cathode voltage. The curve of Fibers shows the emission from a tip on which CNT fibers of about $12 \mathrm{~nm}$ in diameter synthesized by arc discharging and collected have been pasted on filaments. The current more than $100 \mathrm{nA}$ is stably extracted from Tip- 1 at $2.3 \mathrm{kV}$ and $150 \mathrm{nA}$ from Tip-2 at $3.2 \mathrm{kV}$.

Their F-N plots are shown in FIG.4. Assuming the work function is the same for all the tips, the slope of each line is proportional to the radius of the tip. The radius is estimated to be $10 \mathrm{~nm}$ for Tip-1 and $9 \mathrm{~nm}$ for Tip-2. This results shows the emitted area may not be a single tube but a bundle of around ten tubes.

In conclusion, as aligned carbon nanotubes have been produced, the tip area of the CNT has been formed to a $0.3 \mu \mathrm{m} \times 0.3 \mu \mathrm{m}$ square while maintaining the smoothness of the $\operatorname{SiC}(000-1)$ surface.

Other field emission properties (brightness, stability, life-time et al.) will be presented herein. 
Authors would like to acknowledge Mr. A. Okamoto, Toyota Central R\&D Labs, Inc. for supplying SiC crystals and Mr. K. Miyashita, Mazda Motor Corp. for his experimental support.

[1] Y. Saito, K. Hata and T. Murata, Jpn. J. Appl. Phys., 39, L271 (2000).

[2] M. Kusunoki et al., Appl. Phys. Lett., 77, 531 (2000).

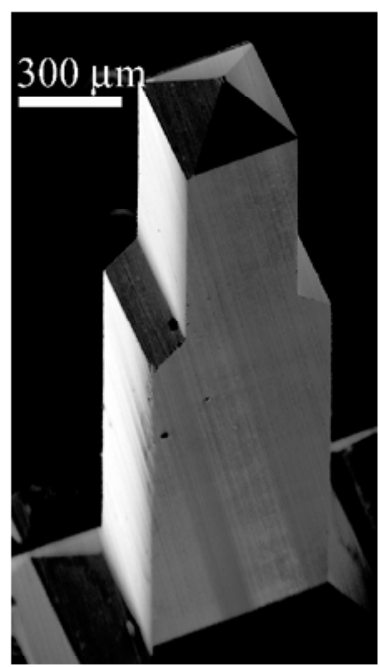

FIG.1 SEM image of SiC tip that was cut out from a SiC single crystal.

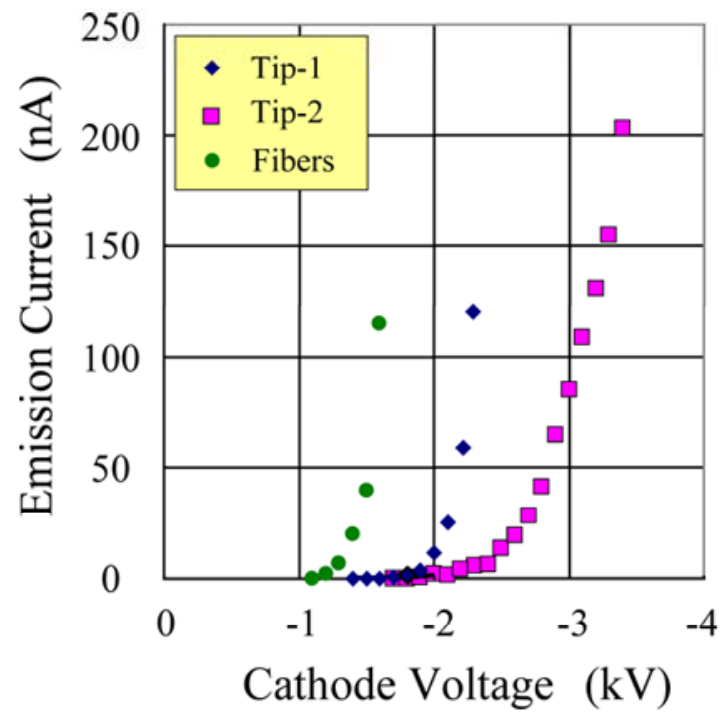

FIG.3 Emission current from CNT tips

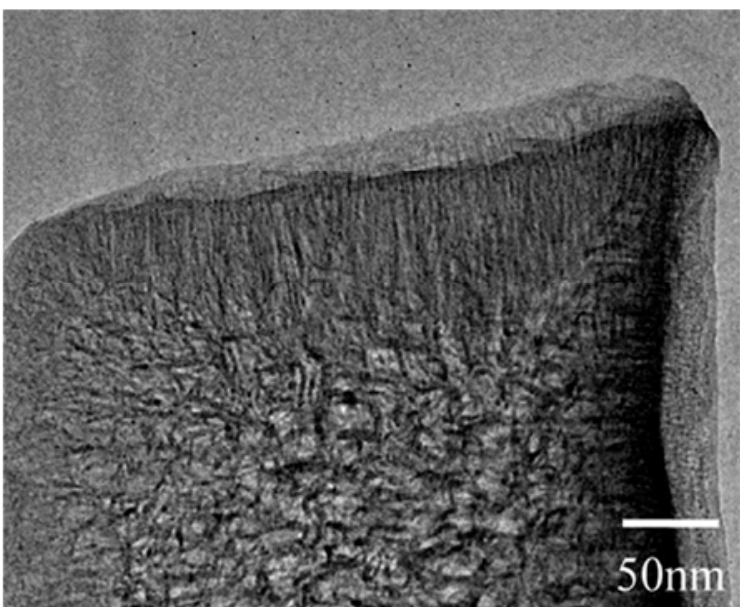

FIG.2 TEM image of the top of the CNT tip. Alined carbon nanotubes are seen perpendicular to the $\mathrm{SiC}(000-1)$ surface.

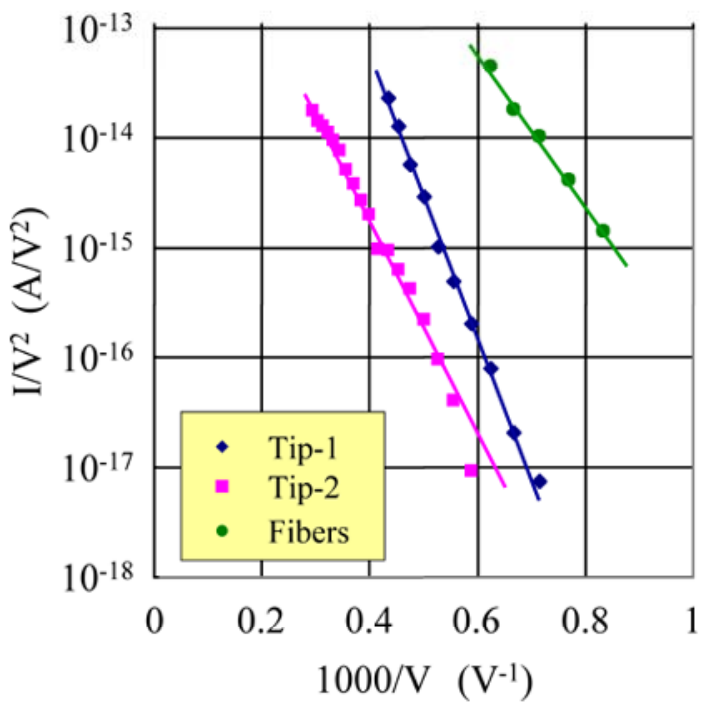

FIG.4 F-N plott of CNT tips 